\title{
SOME NEW ORTHOGONAL DIAGONAL LATIN SQUARES
}

\author{
W. D. WALLIS and L. ZHU
}

(Received 2 November 1981)

Communicated by H. Lausch

\begin{abstract}
A recursive construction for orthogonal diagonal latin squares, using group divisible designs, is presented. In consequence the numbers of orders for which the existence of such squares is in question is reduced to 72 .

1980 Mathematics subject classification (Amer. Math. Soc.): 05 B 15.
\end{abstract}

\section{Introduction}

A latin square of order $v$ is a $v \times v$ array such that every row and every column contains each of the symbols $1,2, \ldots, v$ exactly once. A transversal in a latin square is a set of $v$ positions, one in each row and column, which contain the symbols $1,2, \ldots, v$ in some order. A transversal square is a latin square whose leading diagonal is a transversal, and a diagonal latin square is one whose leading diagonal and back diagonal are both transversals.

Two latin squares $\left(a_{i j}\right)$ and $\left(b_{i j}\right)$ or order $v$ are said to be orthogonal if the $v^{2}$ ordered pairs $\left(a_{i j}, b_{i j}\right)$ include the $v^{2}$ ordered pairs on the symbols $1,2, \ldots, v$ exactly once each. One can then speak of orthogonal transversal squares and orthogonal diagonal latin squares.

For background on latin squares and orthogonality, and for a description of the relation between orthogonal diagonal latin squares and magic squares, the reader should consult [1]. In that book the following problem is posed: for what orders do orthogonal pairs of diagonal latin squares exist? Clearly, 2, 3 and 6 are

c Copyright Australian Mathematical Society 1983 
impossible. In [3] we reduced the set of unsolved orders to 129 members, namely,

$\begin{array}{rrrrrrrrrrrr}10, & 12, & 14, & 15, & 18, & 22, & 24, & 26, & 30, & 34, & 38, & 39, \\ 42, & 46, & 54, & 58, & 60, & 62, & 66, & 70, & 74, & 82, & 86, & 87, \\ 90, & 94, & 98, & 102, & 110, & 114, & 118, & 122, & 126, & 130, & 138, & 142, \\ 146, & 150, & 154, & 158, & 170, & 174, & 178, & 182, & 186, & 194, & 198, & 202, \\ 206, & 214, & 222, & 226, & 230, & 234, & 238, & 242, & 254, & 258, & 262, & 266, \\ 270, & 278, & 282, & 290, & 294, & 306, & 310, & 314, & 318, & 322, & 326, & 327, \\ 334, & 338, & 354, & 366, & 370, & 374, & 378, & 382, & 394, & 398, & 402, & 410, \\ 418, & 422, & 426, & 434, & 446, & 458, & 482, & 490, & 494, & 506, & 522, & 530, \\ 538, & 542, & 566, & 570, & 578, & 586, & 594, & 602, & 614, & 618, & 626, & 634, \\ 642, & 658, & 662, & 674, & 682, & 686, & 690, & 698, & 710, & 734, & 758, & 762, \\ 786, & 878, & 882, & 902, & 926, & 930, & 978, & 1026, & 1074, & & & \end{array}$

(For details and a bibliography of earlier studies, see [3].)

In the present paper we deduce we reduce the above list somewhat by a construction involving group divisible designs.

\section{Group divisible designs}

By a group divisible design $(\mathcal{T} ; \mathcal{G} ; \mathcal{K}$ ) is meant a partition $\mathcal{G}$ (whose parts are called groups) of a finite set $\uparrow$ together with a collection $\mathscr{K}$ of subsets (called blocks) of $\Psi$, such that two members of different groups occur together in precisely one block and two members of the same group do not occur together in any block. If $G$ and $K$ are sets of positive integers such that all group-sizes are in $G$ and all block-sizes are in $K$, and if $v=|\mathscr{q}|$, then $(\mathscr{\Upsilon} ; \mathscr{G} ; \mathcal{K})$ is called a $G D(v ; G ; K)$; if $G$ has only one member $g$, then one writes $G D(v, g, K)$, and similarly if $K$ is singleton.

THEOREM 1. If there exists a set of $k-2$ pairwise orthogonal latin squares of side $g$ then there exists a $G D(g k ; g ; k)$.

PROOF. Suppose the $r$-th latin square has $(i, j)$ entry $l_{i j r}$. Then the blocks

$$
\left\{l_{i j 1}^{1}, l_{i j 2}^{2}, \ldots, l_{i j(k-2)}^{k-2}, i^{k-1}, j^{k}\right\}
$$

for $1 \leqslant i \leqslant g, 1 \leqslant j \leqslant g$, form a $G D(g k ; g ; k)$ whose $r$-th group is $\left\{1^{r}, 2^{r}, \ldots, g^{r}\right\}$.

Various generalizations of this easy result can be obtained; for example, one could delete one block completely, delete all but one element from one block. delete some elements from one group, and so on.

The reader will be familiar with balanced incomplete block designs, or BIBDs. The following construction is useful because BIBDs have been very widely studied. 
THEOREM 2. If there exists a balanced incomplete block design with parameters $(v, b, r, k, 1)$ then there exists $a G D(v-1 ; k-1 ; k)$.

Proof. One treatment is deleted from the balanced incomplete block design. The blocks which previously contained it become the groups of the new group divisible design, and the other blocks become the blocks of the new design.

\section{A construction of orthogonal diagonal latin squares from group divisible designs}

THeOREM 3. Suppose there exists a $G D(v, G, K)$ in which at most one group has odd order. Suppose further that there exist orthogonal diagonal latin squares of order $g$ for all $g \in G$, and there exist orthogonal transversal squares of order $k$ for all $k \in K$. Then there exist orthogonal diagonal latin squares of order $v$.

Proof. First we order the groups in some way, so that no group except (possibly) the last has an odd number of elements. Say they have $g_{1}, g_{2}, \ldots, g_{n}$ elements respectively. Now relabel the elements of the group divisible design so that the first group becomes $\left\{1, v, 2, v-1, \ldots, \frac{1}{2} g_{1}, v-\frac{1}{2} g_{1}+1\right\}$, the second group becomes $\left\{\frac{1}{2} g_{1}+1, v-\frac{1}{2} g_{1}, \frac{1}{2} g_{1}+2, \ldots, v-\frac{1}{2} g_{1}-\frac{1}{2} g_{2}+1\right\}$, and so on.

Next we construct two latin squares $L_{1}$ and $L_{2}$ of size $v$. Given any block $B=\left\{b_{1}, b_{2}, \ldots, b_{k}\right\}$ of size $k$, select two orthogonal transversal squares $H_{1}$ and $H_{2}$ of order $k$ which have common diagonal $(1,2, \ldots, k)$. Define $H_{1}^{*}$ from $H_{1}$ by replacing entry $i$ by $b_{i}$ at every occurrence, for $i=1,2, \ldots, k$. Then the $\left(b_{i}, b_{j}\right)$ entry of $L_{1}$ is set to be the $(i, j)$ entry of $H_{1}^{*} . H_{2}^{*}$ is defined similarly and used to define elements of $L_{2}$. This process is carried out for every block. Next, given any group of size $g$, choose two orthogonal diagonal latin squares $H_{1}$ and $H_{2}$ of side $g$ which have common diagonal $(1,2, \ldots, g)$, and proceed similarly.

Since every pair in $\{1,2, \ldots, v\}$ occurs either in exactly one block or in exactly one group, but not both, this process unambiguously defines $v \times v$ arrays $L_{1}$ and $L_{2}$, which are easily seen to be orthogonal diagonal latin squares, as required.

\section{Applications of the preceding construction}

LEмма 4.1. There exist orthogonal diagonal latin squares of orders 39, 87 and 327. 
Proof. Since there exist three pairwise orthogonal latin squares of all orders except 2, 3, 6, 10 and 14 [4], there exist $G D(5 g ; g ; 5)$ for $g=8,20$ and 80 . So, by deleting some elements from one group, we can construct a $G D\left(4 g_{1}+g_{2} ; g_{1}, g_{2} ; 4,5\right)$ with one group of size $g_{2}$ in the following cases:

$$
\begin{array}{lll}
g_{1}=8, & g_{2}=7, & 4 g_{1}+g_{2}=39 ; \\
g_{1}=20, & g_{2}=7, & 4 g_{1}+g_{2}=87 ; \\
g_{1}=80, & g_{2}=7, & 4 g_{1}+g_{2}=327 .
\end{array}
$$

Now Theorem 3 applies.

REMARK. This method can be used to produce easy solutions of many other odd orders. For example, orders 219, 267, 303, 447 and 543 were solved in [3] by a method involving the construction of several vectors, either by hand computation or computer. They now follow easily, using

$$
\begin{array}{lll}
g_{1}=48, & g_{2}=27, & 4 g_{1}+g_{2}=219 ; \\
g_{1}=56, & g_{2}=43, & 4 g_{1}+g_{2}=267 ; \\
g_{1}=68, & g_{2}=31, & 4 g_{1}+g_{2}=303 ; \\
g_{1}=100, & g_{2}=57, & 4 g_{1}+g_{2}=447 ; \\
g_{1}=120, & g_{2}=63, & 4 g_{1}+g_{2}=543 .
\end{array}
$$

LEMMA 4.2. There exist orthogonal diagonal latin squares of orders 24 and 60.

Proof. It is shown in [2] that a balanced incomplete block design with $k=5$ and $\lambda=1$ exists whenever $v \equiv 1$ or $v \equiv 5(\bmod 20)$. So by Theorem 2 there exists a $G D(v ; 4 ; 5)$ when $v \equiv 0$ or $4(\bmod 20)$. The result then follows from Theorem 3 .

Note that the existence of orthogonal diagonal latin squares of order 24 implies the existence of such squares of order $254=11(24-1)+1$, by Preliminary Theorem 4 or 5 of [3].

LEMMA 4.3. There exist orthogonal diagonal latin squares of order $16 n+d$ whenever $4 n \geqslant d$, when $d=50,78,106$ or 134 .

Proof. There exist three pairwise orthogonal latin squares of order $4 n$ in all the cases concerned, so by Theorem 1 there is a $G D(20 n ; 4 n ; 5)$. So for each value of $d$ we can obtain a $G D(16 n+d ;\{4 n, d\} ;\{5,4\})$ by deleting $4 n-d$ elements from each block, whenever $4 n \geqslant d$. Since orthogonal diagonal latin squares of orders $4 n$ and $d$ exist in every case, orthogonal latin squares of order $16 n+d$ exist. 
From Lemma 4.3 we see that there exist orthogonal diagonal latin squares of order $v$ where $v \equiv 2(\bmod 4)$ and $v \geqslant 666$, and there also exist such squares of orders

$\begin{array}{lllllllll}258, & 290, & 306, & 322, & 338, & 354, & 370, & 398, & 402, \\ 418, & 434, & 446, & 482, & 494, & 530, & 538, & 542, & 570, \\ 578, & 586, & 594, & 602, & 618, & 626, & 634, & 642, & 658 .\end{array}$

To improve this list we need the following specialised construction of group divisible designs.

LEMMA 4.4. If $p$ is a prime power, then there exists a $G D(v ; G ; K)$ with

$$
\begin{aligned}
v & =(k-2)(p-1)+d+e, \\
G & =\{p-1, d, e\}, \\
K & =\{k, k-1, k-2, k-3\}
\end{aligned}
$$

where $k \leqslant p-1, d \leqslant p$ and $e \leqslant p-1$.

Proof. Since $p$ is a prime power and $k \leqslant p-1$, there exist $k$ pairwise orthogonal latin squares of order $p$. So a $G D(p ; k ; p ; k)$ can be constructed using Theorem 1.

Delete all the elements of one block except for the element in group 1. Then delete $p-d$ elements from group 1 and $p-1-e$ elements from another group. The resulting design has the required parameters.

COROLlary. There exist orthogonal diagonal latin squares of orders 490, 506, $522,566,614$ and 662 .

Proof. This follows from Lemma 4.4 and Theorem 3, using the identities

$$
\begin{aligned}
& 490=8 \times 52+50+24 \\
& 506=8 \times 52+50+40 \\
& 522=9 \times 52+50+4 \\
& 566=9 \times 52+50+48 \\
& 614=9 \times 60+50+24 \\
& 662=8 \times 72+50+36 .
\end{aligned}
$$


Summarizing the results of this section we have

THEOREM 4. There exist orthogonal diagonal latin squares of every order $v$ where $v>458$. Orders 2, 3 and 6 are impossible; the only orders for which the existence is undecided are:

$10, \quad 12, \quad 14, \quad 15, \quad 18, \quad 22, \quad 26, \quad 30, \quad 34, \quad 38, \quad 42, \quad 46$,

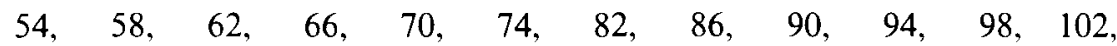

$110, \quad 114,118, \quad 122,126,130,138,142, \quad 146, \quad 150, \quad 154,158$,

$170,174,178, \quad 182,186,194,198,202, \quad 206,214, \quad 222, \quad 226$,

$230, \quad 234, \quad 238, \quad 242, \quad 262, \quad 266, \quad 270, \quad 278, \quad 282, \quad 294, \quad 310, \quad 314$,

$318,326,334,366,374,378, \quad 382, \quad 394,410,422, \quad 426,458$.

\section{References}

[1] J. Denes and A. D. Keedwell, Latin squares and their applications (Academic Press, New York. 1974).

[2] H. Hanani, 'Balanced incomplete block designs and related designs', Discrete Math. 11 (1975), 255-369.

[3] W. D. Wallis and L. Zhu, 'Existence of orthogonal diagonal latin squares', Ars Combinatoria 12 (1981). 51-68.

[4] S. M. P. Wang and R. M. Wilson, 'A few more squares, II', Congressus Numerantium 21 ( $=$ Proceedings of the Ninth Southeastern Conference on Combinatorics, Graph Theory and Computing), 688 .

Department of Mathematics

The University of Newcastle

Newcastle, N.S.W. 2308

Australia
Department of Mathematics

Kiangsu Teachers' College

Soochow

China 